\title{
EFFICACY OF DIFFERENT SAMPLING METHODS IN PEST MONITORING OF DIRECT SEEDED RICE AT DIFFERENT GROWTH STAGES
}

\author{
DUMAN, M. ${ }^{1 *}-$ MUTLU, Ç. ${ }^{2}$ \\ ${ }^{I}$ Diyarbakır Plant Protection Research Institute, 21100 Sur, Diyarbakir, Turkey \\ ${ }^{2}$ Department of Plant Protection, Faculty of Agriculture, Harran University, Sanliurfa, Turkey \\ ${ }^{*}$ Corresponding author \\ email: cetinmutlu21@hotmail.com; phone: +90-414-318-1248; fax: +90-414-318-3274
}

(Received $17^{\text {th }}$ Apr 2019; accepted $13^{\text {th }}$ Jun 2019)

\begin{abstract}
This two-year study was aimed at determining the efficacy of different sampling methods in pest monitoring of direct seeded rice in southeastern Anatolia, Turkey. Four direct seeded rice fields were selected in Sanliurfa and Diyarbakir provinces and insect species were monitored at four different BBCH rice growth stages (i.e., 21-39, 41-49, 51-69 and 71-89) with two different sampling methods (D-Vac and sweep net). The highest density of insects belonging to Tipulidae family was observed at Biologische Bundesanstalt, Bundessortenamt and Chemical industry $(\mathrm{BBCH}) 21-39$ growth stage the both years of the study. Similarly, the highest density of Phloeothripidae species was noted during BBCH 71-89 growth stage during 1st year and BBCH 21-39 growth stage during 2nd year of the study. Likewise, the highest density for Tettigonidae insects was recorded at $\mathrm{BBCH}$ 51-69 growth stage during both years. The greatest density of Acrididae family was recorded at BBCH 41-649 growth stage during both years of study. Overall, D-Vac sampling method trapped higher number of insects belonging to Tipulidae and Acrididae families, whereas sweep net method trapped higher number of insects belonging to Tettigonidae and Phloeothripidae families. It is concluded that D-Vac sampling method can be used for sampling Tipulidae and Acrididae insect species infesting rice in the future studies.
\end{abstract}

Keywords: Tipulidae, Phloeothripidae, Tettigonidae, D-Vac, Turkey

\section{Introduction}

Rice provides dietary and caloric supplies for half of the population of the world (Muthayya et al., 2014). Globally rice is cultivated on 167 million hectares of land with an annual production of 769 million tons and an average yield of $4601 \mathrm{~kg} \mathrm{ha}^{-1}$. In Turkey, rice is grown on 109 thousand hectares with an annual production of 900 thousand tones with an average yield of $8218 \mathrm{~kg} \mathrm{ha}^{-1}$ (FAOSTAT, 2019). In the Southeastern Anatolia Region of Turkey, rice occupies 1965 hectares, producing 9 thousand tones with an average yield of $4580 \mathrm{~kg} \mathrm{ha}^{-1}$ (TURKSTAT, 2016). Turkey is among the top five countries in terms of average rice yield (FAOSTAT, 2019), yet there exists a yield gap compared to developed countries of the world, i.e., Australia and the United States. Rice cultivation faces various limitations ranging from climate, soil type and fertility, water supply, irrigation, pest and disease problems in Turkey.

Rice farming is gaining popularity among Turkish farmers due to higher demands and government support policies to increase production for gaining self-sufficiency in rice production (Duman and Mutlu, 2011; Duman et al., 2013, 2014; Mutlu et al., 2016). The increasing rice cultivation has given rise to several problems such as insect, disease and weed' infestation, which hamper the quantity and quality of rice. Several studies have determined the beneficial and harmful insect fauna in rice cultivation areas of 
Aegean, Black Sea and Marmara regions in Turkey (Uzunali, 1976; Teoman and Kavut, 1979; Yürüten et al., 1982).

Transplanted rice fields are considered as the most diverse agricultural systems on the planet (Horgan, 2017). The rice productivity depends upon the flood cycles in transplanted rice where periodic shifts are recorded from flooded to dry period during the crop cycle. These changes in flood cycles promote a rich diversity of flora and fauna in transplanted rice (Catling, 1992; Way and Heong, 1994; Settle et al., 1996; Horgan et al., 2017). Over 800 insects have been recorded to feed on rice; however, 20 of these species are considered as damaging pests of rice crop (Castro, 1970; Pathak and Dyck, 1973; Kiritani, 1979; Pathak and Khan, 1994). The most damaging pest species of rice include stem borers, gall midge, defoliators and vectors such as leaf and plant hoppers that cause direct damages and transmit various diseases. These pest species incur heavy losses to rice crop and various management strategies are opted or devised to keep their level below economic threshold. China, the largest rice producer has developed enough genomic strategies, which keep the harmful pests below their threshold level, whereas rice producers in the rest of the rice producing countries are still dependent on conventional pest management strategies.

Turkey is among the countries where farmers are still dependent on conventional pest management strategies for rice crop. The pest management studies in rice are mostly concentrated on the identification of beneficial and harmful pest species. Most of these studies have been conducted in transplanted rice, whereas direct seeded rice has been less studied (Duman and Mutlu, 2011; Duman et al., 2013, 2014; Mutlu et al., 2016). Rice is sown through direct seeding method in southeastern Anatolia region and scanty information is available on the pests of direct seeded rice crop in the region. Moreover, no information is available regarding the pest species' infestation at different growth stages of direct seeded rice in southeastern Anatolia region. Various studies in transplanted rice crop has revealed that relatively small number of arthropod herbivores damage rice plants; however, most arthropods species are beneficial. Herbivores invade the rice fields as crop grows and a small number of these can become pests if not effectively regulated. The pest attack at early crop growth stage relatively less damaging and negligible yield losses happen (Rubia et al., 1996; Horgan et al., 2016) or in some cases early stage pest attack results in more yield compared to pest attack-free crop (Nik Mohammad Noor et al., 1995; Islam and Karim, 1997). However, the pest attack at later growth stages can be more damaging and could result in heavy economic losses (Horgan, 2017). Therefore, monitoring pest density at different growth stages is necessary for effective pest management in rice crop (Reissig et al., 1986).

Different sampling methods are used to monitor pest densities in different crops. The sweep net and D-Vac sampling methods are most frequently used for pest monitoring (Turnipseed, 1974; Reissig et al., 1986; Schotzko and O'Keeffe, 1989; Buffington and Redak, 1998). These sampling methods have differential efficacy for trapping different insect species. The selection of accurate sampling method could help in effective monitoring of insect species (Schotzko and O'Keeffe, 1989; Binns and Nyrop, 1992; Buffington and Redak, 1998) However, limited information is available on the efficacy of insect monitoring in rice crop at different growth stages.

Therefore, the current study was designed with three major objectives, i.e., i) identify the harmful pest species infesting direct seeded rice crop in different districts of southeastern Anatolia region, ii) infer the density of different harmful pest species at different growth stages of rice and iii) determine the efficacy of different sampling 
methods in monitoring insect species at different growth stages of rice crop. It was hypothesized that sampling districts and growth stages will significantly differ for the density of harmful pest species. It was further hypothesized that different sampling methods will differ in their efficacy for monitoring/trapping different insect species. To test these hypotheses, survey studies were conducted during two consecutive years in four districts of southeastern Anatolia region, Turkey. The results of the study will help to devise effective management strategies for the harmful pest species in direct seeded rice and help to improve sampling methods for pest monitoring of rice crop.

\section{Material and Methods}

\section{Study area}

This survey study was conducted in three districts of Diyarbakir province (Ergani, Cinar and Hazro) and one district of Sanliurfa (Siverek) province during rice growing seasons of 2010 and 2011. One rice field was selected from each district and surveys were accomplished in these selected fields during both years of the study. The geographic location of each surveyed field and prevailing long-term climatic conditions are provided in Table 1. Rice is cultivated by direct seeding method in the region. The farmers use different herbicide to manage weed infestation. Penoxulam, BispyribacSodium and Bentazone+MCPA herbicides are used at the initial stage of rice crop for weed management. Insecticides are generally not used to control insect pests of rice crop in southeastern Anatolia region of Turkey.

Table 1. Geographic location and long-term average (1979-2013) weather attributes of surveyed fields in southeastern Anatolia region, Turkey

\begin{tabular}{c|c|c|c|c|c|c|c|c|c|c}
\hline $\begin{array}{c}\text { Surveyed } \\
\text { Field }\end{array}$ & $\begin{array}{c}\text { Latitude } \\
{ }^{\circ} \mathbf{N}\end{array}$ & $\begin{array}{c}\text { Longitude } \\
{ }^{\circ} \mathbf{E}\end{array}$ & $\begin{array}{c}\text { Area } \\
(\mathbf{h a})\end{array}$ & Altitude & $\begin{array}{c}\text { Rainfall } \\
(\mathbf{m m})\end{array}$ & $\begin{array}{c}\text { Max T } \\
{ }^{\circ} \mathbf{C}\end{array}$ & $\begin{array}{c}\text { Min T } \\
{ }^{\circ} \mathbf{C}\end{array}$ & $\begin{array}{c}\text { Avg T } \\
{ }^{\circ} \mathbf{C}\end{array}$ & $\begin{array}{c}\text { Sunshine } \\
\text { Hours }\end{array}$ & $\begin{array}{c}\text { Aridity } \\
\text { Index }\end{array}$ \\
\hline Siverek & 37.710583 & 39.246194 & 10.5 & 630 & 490.43 & 27.66 & 9.55 & 21.07 & 6.32 & 0.39 \\
Ergani & 37.881462 & 39.720773 & 11.0 & 1057 & 658.84 & 26.74 & 7.48 & 18.28 & 6.81 & 0.52 \\
Hazro & 38.168675 & 40.741611 & 14.0 & 821 & 617.05 & 26.22 & 8.27 & 19.61 & 6.72 & 0.52 \\
Cinar & 37.794088 & 40.156301 & 12.0 & 766 & 503.46 & 28.03 & 8.78 & 20.82 & 7.25 & 0.38 \\
\hline
\end{tabular}

Max = Maximum, Min = Minimum, Avg = Average, Aridity index is opted from Çetin et al. (2007)

\section{Survey timing and methods}

Rice is typically cultivated during the month of May in southeastern Anatolia region of Turkey. The exploratory surveys to collect the insect species infesting rice crop were conducted at four different growth stages of rice. The growth stages according to $\mathrm{BBCH}$ scale were; 21-39 (tillering and stem elongation), 41-49 (booting), 51-69 (inflorescence emergence and anthesis) and 71-89 (fruit development and ripening) (Lancashire et al., 1991; Meier, 1997). The surveys were started from mid-June and continued until maturity with a fortnight interval. Two different sampling methods were used to test their efficacy in collecting the insect species. For sweep net method, insects were collected by sweeping the standard sweep net (Yiğit et al., 2003) at four different locations of each field. Twenty-five nets were swept at each location within a field making 100 sweeps per field. Similarly, in the D-Vac method (Mutlu et al., 2008; Y1lmaz and Karsavuran, 2009), insects were sucked from four different locations within 
each sampled field. The insects were sucked for one minute with vacuum sucking. For the sake of uniformity, twenty-five suctions were made at each location within each field. The collected insect species from both sampling methods were put in transparent polyethylene bags, labeled and brought to the laboratory. The collected species were identified according to the earlier specimens in the region, whereas the unidentified specimens were sent to taxonomists for accurate identification. The grasshopper specimens were identified by Prof. Dr. Ali SATAR (Faculty of Science and Literature, Dicle University, Diyarbakir, Turkey). Similarly, thrips specimens were identified by Prof. Dr. İrfan TUNÇ (Department of Plant Protection, Faculty of Agriculture, Akdeniz University, Antalya, Turkey). Crane fly (Tipula orientalis) was identified by Prof. Dr. Hasan KOÇ (Department of Biology, Faculty of Sciences, Muğla Sitkı Koçman University, Muğla, Turkey).

The polyethene bags were kept in deep freezer to kill the insect species collected during the survey. The insect species were then placed over white papers and carefully counted. The counted species were separated into two groups (i.e., beneficial and harmful insects), and data relating to harmful insect species were used in this manuscript. The identified harmful insect species were then grouped into different families. The density of the insect species was then counted at family level and used for the interpretation of the results. A total 13 harmful insect species belonging to 4 families, i.e., Tipulidae (1 species), Acrididae ( 8 species), Tettigonidae (2 species) and Phloeothripidae ( 2 species) were identified during the survey.

\section{Statistical analyses}

The collected data regarding the density of insect species in different families were tested for normality, which indicated a normal distribution. Therefore, the statistical analyses were performed on non-transformed, original data. A paired t test was used to infer the differences among years, which indicated significant differences. Therefore, the data of both study years were analyzed and interpreted separately. Three-way Analysis of variance (ANOVA) technique was used to infer the effect of different growth stages, sampling methods and surveyed fields on the density of insect species belonging to different families (Steel et al., 1997). Least significant difference test at 99\% probability level was used as post-hoc test to separate the means where ANOVA indicated significance. ANOVA indicated that all individual and interactive effects of growth stage, sampling method and surveyed field were significant for all insect families. Therefore, three-way interactions, i.e., growth stage $\times$ sampling method $\times$ surveyed fields were used for the interpretation of the results. All statistical analyses were performed using SPSS Version 20.0 (IBM, 2012).

\section{Results}

A total of 2472 insects belonging to 4 families were collected during 2010, whereas 3104 insects belonging to the same families were collected during 2011. The highest percentage (45.28\%) of the insects belonged to Tettigonidae family, whereas the lowest percentage $(8.09 \%)$ of the collected insect species belonged to Acrididae family (Figure 1).

The identified insect species with their corresponding families are summarized in Table 2. Some of the identified insect species are shown in Figure 2. 
The individual and interactive effects of growth stage, sampling methods and surveyed fields significantly influenced the density of Tipulidae insect species during both years of the study, with an exception of non-significant effect of sampling methods $\times$ surveyed fields during 2011 (Table 3).

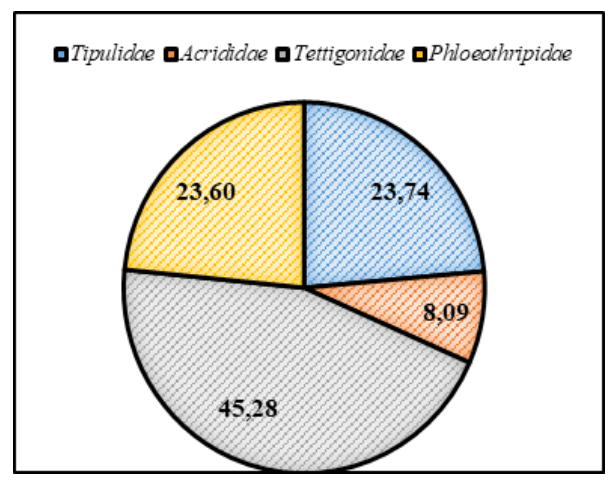

Figure 1. The percentage of different harmful insect species belonging to various families collected from rice fields in southeastern Anatolia region, Turkey during 2010 and 2011

Table 2. Harmful insect species identified in different rice fields surveyed in southeastern Anatolia region, Turkey during 2010 and 2011

\begin{tabular}{|c|c|c|}
\hline Order & Family & Species \\
\hline Diptera & Tipulidae & Tipula orientalis Lackschewitz \\
\hline Orthoptera & Acrididae & Aiolopus strepens (Latr.) \\
\hline Orthoptera & Acrididae & Platycleis escalerai Bolívar \\
\hline Orthoptera & Acrididae & Heteracris pterosticha (Fischer von Waldheim) \\
\hline Orthoptera & Acrididae & Thisoicetrinus pterostichus (Fischer de Waldheim) \\
\hline Orthoptera & Acrididae & Calliptamus barbarus Fisher-Waldheim \\
\hline Orthoptera & Acrididae & Euprepocnemis plorans Charp. \\
\hline Orthoptera & Acrididae & Oedipoda aurea Uvarov \\
\hline Orthoptera & Acrididae & Aiolopus strepens (Latreille) \\
\hline Orthoptera & Tettigonidae & Isophya sp. \\
\hline Orthoptera & Tettigonidae & Conocephalus fuscus (Fabricius) \\
\hline Thysanoptera & Phloeothripidae & Haplothrips aculeatus (Fabr.) \\
\hline Thysanoptera & Phloeothripidae & Haplothrips reuteri (Karny) \\
\hline
\end{tabular}

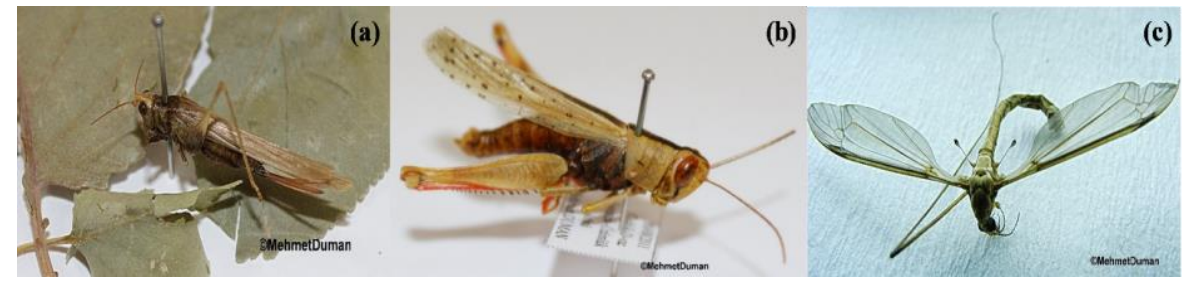

Figure 2. Different hamrful insect species, (a) Conocephalus fuscus Fabricius (Tettigoniidae), (b) Thisoicetrinus pterostichus F.W. (Catantopidae) and (c) Tipula orientalis Lackschewitz identified from rice fields in southeastern Anatolia region, Turkey during 2010 and 2011

The highest density of Tipulidae insect species during 2010 was recorded at $\mathrm{BBCH}$ 21-39 growth stage in Siverek field with D-Vac sampling method, whereas the lowest density was noted at BBCH 51-69 and BBCH 71-89 growth stages in Hazro and Cinar fields with D-Vac sampling method (Table 4). Similarly, D-Vac sampling method 
collected the highest number of Tipulidae insect species at BBCH 21-39 growth stage from Cinar field during 2011, while the lowest number of insects were trapped by sweep net sampling method from Cinar field at BBCH 41-49 growth stage of rice (Table 4). Overall, D-Vac sampling method trapped higher number of Tipulidae insect species compared with sweep net sampling method during both years of the study. Siverek field had the higher density of Tipulidae insect species during both years of the study compared to the rest of the sampling fields (Table 4).

Table 3. Three-way Analysis of variance of different growth stages, sampling methods, surveyed fields and their interaction for the number of individuals trapped belonging to different insect families

\begin{tabular}{|c|c|c|c|c|c|c|c|c|c|}
\hline \multicolumn{10}{|c|}{2010} \\
\hline \multirow{2}{*}{ Source of Variation } & & \multicolumn{2}{|c|}{$\begin{array}{l}\text { Tipulidae } \\
\end{array}$} & \multicolumn{2}{|c|}{ Phloeothripidae } & \multicolumn{2}{|c|}{ Tettigonidae } & \multicolumn{2}{|c|}{ Acrididae } \\
\hline & df & F Value & P Value & F Value & P Value & F Value & P Value & F Value & P Value \\
\hline Growth Stage (G) & 3 & 790.62 & $0.0001 *$ & 161.41 & $0.0001 *$ & 331.91 & $0.0001 *$ & 369.33 & $0.0001 *$ \\
\hline Sampling Method (M) & 1 & 741.23 & $0.0001 *$ & 738.44 & $0.0001 *$ & 82.39 & $0.0001 *$ & 4.17 & $0.0452^{\mathrm{NS}}$ \\
\hline Surveyed Field (F) & 3 & 1502.22 & $0.0001 *$ & 1636.76 & $0.0001 *$ & 721.70 & $0.0001 *$ & 251.75 & $0.0001 *$ \\
\hline $\mathrm{G} \times \mathrm{M}$ & 3 & 254.46 & $0.0001 *$ & 434.20 & $0.0001 *$ & 36.80 & $0.0001 *$ & 58.15 & $0.0001 *$ \\
\hline $\mathrm{G} \times \mathrm{F}$ & 9 & 277.14 & $0.0001 *$ & 184.07 & $0.0001 *$ & 59.52 & $0.0001 *$ & 117.51 & $0.0001 *$ \\
\hline $\mathrm{M} \times \mathrm{F}$ & 3 & 55.14 & $0.0001 *$ & 78.26 & $0.0001 *$ & 7.17 & $0.0003 *$ & 39.51 & $0.0001 *$ \\
\hline $\mathrm{G} \times \mathrm{M} \times \mathrm{F}$ & 9 & 121.17 & $0.0001 *$ & 166.06 & $0.0001 *$ & 52.76 & $0.0001 *$ & 53.30 & $0.0001 *$ \\
\hline \multicolumn{10}{|c|}{2011} \\
\hline Growth Stage (G) & 3 & 9050.79 & $0.0001 *$ & 1179.58 & $0.0001 *$ & 8379.90 & $0.0001 *$ & 844.30 & $0.0001 *$ \\
\hline Sampling Method (M) & 1 & 1635.57 & $0.0001 *$ & 2.50 & $0.12^{\mathrm{NS}}$ & 426.02 & $0.0001 *$ & 217.27 & $0.0001 *$ \\
\hline Surveyed Field (F) & 3 & 39.21 & $0.0001 *$ & 12.78 & $0.0001 *$ & 13.49 & $0.0001 *$ & 2.36 & $0.08^{\mathrm{NS}}$ \\
\hline $\mathrm{G} \times \mathrm{M}$ & 3 & 992.07 & $0.0001 *$ & 939.62 & $0.0001 *$ & 1243.95 & $0.0001 *$ & 740.73 & $0.0001 *$ \\
\hline $\mathrm{G} \times \mathrm{F}$ & 9 & 22.00 & $0.0001 *$ & 8.22 & $0.0001 *$ & 21.36 & $0.0001 *$ & 2.57 & $0.01 *$ \\
\hline $\mathrm{M} \times \mathrm{F}$ & 3 & 0.55 & $0.65^{\mathrm{NS}}$ & 8.05 & $0.0001 *$ & 5.90 & $0.0001 *$ & 5.31 & $0.0001 *$ \\
\hline $\mathrm{G} \times \mathrm{M} \times \mathrm{F}$ & 9 & 23.33 & $0.0001 *$ & 5.70 & $0.0001 *$ & 10.47 & $0.0001 *$ & 4.89 & $0.0001 *$ \\
\hline
\end{tabular}

$\mathrm{df}=$ degree of freedom, $*=$ significant at $\mathrm{P} \leq 0.01, \mathrm{NS}=$ non-significant

Table 4. Total number of individuals belonging to Tipulidae family trapped by two different sampling methods at different growth stages of rice from southeastern Anatolia region, Turkey during 2010 and 2011

\begin{tabular}{|c|c|c|c|c|c|c|c|c|}
\hline \multicolumn{9}{|c|}{2010} \\
\hline \multirow{2}{*}{ Growth stage } & \multicolumn{4}{|c|}{ D-Vac } & \multicolumn{4}{|c|}{ Sweep net } \\
\hline & Siverek & Ergani & Hazro & Çinar & Siverek & Ergani & Hazro & Çinar \\
\hline BBCH 21-39 & $76.33 \mathrm{a}$ & $16.00 \mathrm{ijk}$ & $15.00 \mathrm{jk}$ & 9.00 no & $50.00 \mathrm{c}$ & $32.33 \mathrm{e}$ & $19.00 \mathrm{gh}$ & $12.00 \mathrm{~lm}$ \\
\hline ВBCH 41-49 & 8.00 no & $30.00 \mathrm{e}$ & $5.00 \mathrm{pq}$ & $5.00 \mathrm{pq}$ & $41.33 \mathrm{~d}$ & $60.33 \mathrm{~b}$ & $21.00 \mathrm{~g}$ & 9.00 no \\
\hline ВBCH 51-69 & $10.67 \mathrm{mn}$ & $12.00 \mathrm{~lm}$ & $1.00 \mathrm{r}$ & $1.00 \mathrm{r}$ & $47.67 \mathrm{c}$ & $24.00 \mathrm{f}$ & $1.00 \mathrm{r}$ & $14.00 \mathrm{kl}$ \\
\hline ВBCH 71-89 & $18.00 \mathrm{hi}$ & $7.00 \mathrm{op}$ & $1.00 \mathrm{r}$ & $1.00 \mathrm{r}$ & $17.00 \mathrm{hi}$ & $10.33 \mathrm{mn}$ & $1.00 \mathrm{r}$ & $2.33 \mathrm{qr}$ \\
\hline LSD 0.01 & \multicolumn{8}{|c|}{2.68} \\
\hline \multicolumn{9}{|c|}{2011} \\
\hline ВBCH 21-39 & $79.00 \mathrm{a}$ & $69.67 \mathrm{~b}$ & $65.00 \mathrm{c}$ & $66.67 \mathrm{c}$ & $44.00 \mathrm{~d}$ & $36.33 \mathrm{f}$ & $41.00 \mathrm{e}$ & $40.33 \mathrm{e}$ \\
\hline ВBCH 41-49 & $10.00 \mathrm{~lm}$ & $10.00 \mathrm{~lm}$ & $9.00 \mathrm{~m}$ & $10.00 \mathrm{~lm}$ & $10.00 \mathrm{~lm}$ & $9.00 \mathrm{~m}$ & $9.00 \mathrm{~m}$ & $6.00 \mathrm{n}$ \\
\hline ВBCH 51-69 & $19.00 \mathrm{gh}$ & $15.00 \mathrm{i}$ & $19.00 \mathrm{gh}$ & $14.00 \mathrm{jk}$ & $14.00 \mathrm{jk}$ & $14.00 \mathrm{jk}$ & 11.001 & $13.00 \mathrm{k}$ \\
\hline ВBCH 71-89 & $15.00 \mathrm{i}$ & $20.00 \mathrm{~g}$ & $19.00 \mathrm{gh}$ & $19.00 \mathrm{gh}$ & $19.00 \mathrm{gh}$ & $18.00 \mathrm{~h}$ & $16.00 \mathrm{i}$ & $16.00 \mathrm{i}$ \\
\hline LSD 0.01 & \multicolumn{8}{|c|}{1.76} \\
\hline
\end{tabular}

Means sharing the same letters within a column or a row are statistically similar $(P>0.01)$

The density of insect species belonging to Phloeothripidae family was significantly altered by the individual and interactive effects of growth stage, sampling methods and surveyed fields, with only a non-significant effect of sampling methods 2011 (Table 3). 
The highest and the lowest number of Phloeothripidae insect species were trapped by sweep net sampling method at BBCH 71-89 growth stage from Cinar and Siverek fields, respectively during 2010 (Table 5). However, the highest number of insect species belonging to Phloeothripidae family were trapped by D-Vac sampling method at BBCH 21-39 growth stage from Ergani field and by sweep net sampling method at BBCH 51-69 growth stage from Siverek field during 2011. The lowest number of Phloeothripidae insect species during 2011 were collected by sweep net sampling method at BBCH 71-89 growth stage from all the sampling fields included in the study (Table 5). The sweep net sampling method exhibited higher efficacy in trapping Phloeothripidae insect species during the first year, while both sampling method indicated similar performance during the second year of the study (Table 5).

Table 5. Total number of individuals belonging to Phloeothripidae family trapped by two different sampling methods at different growth stages of rice from southeastern Anatolia region, Turkey during 2010 and 2011

\begin{tabular}{|c|c|c|c|c|c|c|c|c|}
\hline \multicolumn{9}{|c|}{2010} \\
\hline \multirow{2}{*}{ Growth stage } & \multicolumn{4}{|c|}{ D-Vac } & \multicolumn{4}{|c|}{ Sweepnet } \\
\hline & Siverek & Ergani & Hazro & Çinar & Siverek & Ergani & Hazro & Çinar \\
\hline BBCH 21-39 & $33.00 \mathrm{gh}$ & $17.67 \mathrm{mn}$ & $23.00 \mathrm{i}$ & 36.00 ef & $15.00 \mathrm{op}$ & $21.00 \mathrm{jk}$ & $35.00 \mathrm{fg}$ & $24.00 \mathrm{i}$ \\
\hline ВВCH 41-49 & $20.33 \mathrm{kl}$ & $14.00 \mathrm{p}$ & 16.33 no & $25.00 \mathrm{i}$ & $19.00 \mathrm{klm}$ & $31.67 \mathrm{~h}$ & $49.00 \mathrm{~b}$ & $52.33 \mathrm{a}$ \\
\hline ВBCH 51-69 & $10.00 \mathrm{q}$ & $7.00 \mathrm{rs}$ & $19.00 \mathrm{klm}$ & $38.00 \mathrm{de}$ & $35.00 \mathrm{fg}$ & $21.00 \mathrm{jk}$ & $34.00 \mathrm{fg}$ & $41.00 \mathrm{c}$ \\
\hline BBCH 71-89 & $5.00 \mathrm{st}$ & $3.00 \mathrm{tu}$ & $35.00 \mathrm{fg}$ & $39.67 \mathrm{~cd}$ & $1.00 \mathrm{u}$ & $8.00 \mathrm{qr}$ & $18.33 \mathrm{lmn}$ & $52.67 \mathrm{a}$ \\
\hline LSD 0.01 & \multicolumn{8}{|c|}{2.13} \\
\hline \multicolumn{9}{|c|}{2011} \\
\hline BBCH 21-39 & $32.33 \mathrm{ab}$ & $34.00 \mathrm{a}$ & $32.00 \mathrm{ab}$ & $29.00 \mathrm{~cd}$ & $12.67 \mathrm{i}$ & $14.00 \mathrm{hi}$ & $14.00 \mathrm{hi}$ & $13.00 \mathrm{hi}$ \\
\hline ВBCH 41-49 & $22.00 \mathrm{e}$ & $21.00 \mathrm{ef}$ & $14.00 \mathrm{hi}$ & $14.00 \mathrm{hi}$ & $19.00 \mathrm{fg}$ & $15.00 \mathrm{~h}$ & $18.00 \mathrm{~g}$ & $15.00 \mathrm{~h}$ \\
\hline ВBCH 51-69 & $10.00 \mathrm{k}$ & $9.00 \mathrm{k}$ & $11.00 \mathrm{jk}$ & $10.00 \mathrm{k}$ & $34.00 \mathrm{a}$ & $28.00 \mathrm{~d}$ & $30.33 \mathrm{bc}$ & $31.00 \mathrm{bc}$ \\
\hline ВBCH 71-89 & 4.331 & 4.001 & 4.001 & 4.001 & $1.00 \mathrm{~m}$ & $1.00 \mathrm{~m}$ & $1.00 \mathrm{~m}$ & $1.00 \mathrm{~m}$ \\
\hline LSD 0.01 & \multicolumn{8}{|c|}{2.10} \\
\hline
\end{tabular}

Means sharing the same letters within a column or a row are statistically similar $(\mathrm{P}>0.01)$

All individual and interactive effects growth stage, sampling methods and surveyed fields significantly affected the density of insect species belonging to Tettigonidae family during both years of study (Table 3). The highest density of insect species belonging to Tettigonidae family was observed at $\mathrm{BBCH}$ 51-69 growth stage from Siverek field during both years of the study (Table 6). The sweep net sampling method collected the highest number of Tettigonidae insect species from Siverek field at BBCH 51-69 growth stage during both survey years. Similarly, the lowest number of insect species belonging to Tettigonidae family were trapped from Ergani field by both sampling methods at BBCH 71-89 growth stage during 2010. However, the lowest number of Tettigonidae insect species were trapped by D-Vac sampling method from Siverek field at BBCH 71-89 growth stage during 2011 (Table 6).

The density of Acrididae insect species belonging to family was significantly altered by all individual and interactive effects of growth stage, sampling methods and surveyed fields during both study years except non-significant effects of sampling methods during 2010 and surveyed fields during 2011 (Table 3). The highest number of Acrididae insect species were recorded at $\mathrm{BBCH}$ 41-49 growth stage of rice during both years of the study (Table 7). The D-Vac sampling method from Siverek field and sweep net sampling method from Cinar field collected the highest number of insects belonging to Acrididae family at $\mathrm{BBCH}$ 41-49 growth stage during first year of the study 
(Table 7). The lowest number of Acrididae insect species were trapped by D-Vac sampling method from Siverek field at BBCH 21-39 growth stage during 2010 (Table 7). During the second year, D-Vac sampling method trapped the highest number of Acrididae insect species at BBCH 41-49 growth stage from Siverek and Cinar fields, whereas the lowest number of Acrididae species were collected by D-Vac sampling method at BBCH 71-89 growth stage from all sampling fields (Table 7).

Table 6. Total number of individuals belonging to Tettigonidae family trapped by two different sampling methods at different growth stages of rice from southeastern Anatolia region, Turkey during 2010 and 2011

\begin{tabular}{|c|c|c|c|c|c|c|c|c|}
\hline \multicolumn{9}{|c|}{2010} \\
\hline \multirow{2}{*}{ Growth stage } & \multicolumn{4}{|c|}{ D-Vac } & \multicolumn{4}{|c|}{ Sweepnet } \\
\hline & Siverek & Ergani & Hazro & Çinar & Siverek & Ergani & Hazro & Çinar \\
\hline ВBCH 21-39 & $60.33 \mathrm{c}$ & $14.00 \mathrm{jkl}$ & $20.00 \mathrm{~h}$ & $10.001-0$ & 39.67 ef & $11.00 \mathrm{k}-\mathrm{m}$ & $14.00 \mathrm{jkl}$ & $30.67 \mathrm{~g}$ \\
\hline ВBCH 41-49 & $59.67 \mathrm{c}$ & $21.00 \mathrm{hi}$ & $25.00 \mathrm{~h}$ & $19.33 \mathrm{i}$ & $68.67 \mathrm{~b}$ & $31.00 \mathrm{ge}$ & $44.00 \mathrm{de}$ & $48.33 \mathrm{~d}$ \\
\hline ВBCH 51-69 & $65.00 \mathrm{bc}$ & 6.67 no & $12.33 \mathrm{klm}$ & $36.00 \mathrm{fg}$ & $99.67 \mathrm{a}$ & $8.00 \mathrm{mno}$ & $38.33 \mathrm{f}$ & $10.001-0$ \\
\hline ВBCH 71-89 & $11.67 \mathrm{k}-\mathrm{m}$ & $0.83 \mathrm{p}$ & $12.00 \mathrm{ijk}$ & $16.00 \mathrm{~g}$ & $30.67 \mathrm{~g}$ & $1.00 \mathrm{p}$ & $5.00 \mathrm{op}$ & $10.331-0$ \\
\hline LSD 0.01 & \multicolumn{8}{|c|}{5.52} \\
\hline \multicolumn{9}{|c|}{2011} \\
\hline BBCH 21-39 & $56.00 \mathrm{~h}$ & $52.33 \mathrm{i}$ & $56.33 \mathrm{~h}$ & $60.33 \mathrm{~g}$ & $38.33 \mathrm{k}$ & $29.00 \mathrm{~m}$ & 33.001 & $31.00 \mathrm{~lm}$ \\
\hline ВBCH 41-49 & $60.33 \mathrm{~g}$ & $60.33 \mathrm{~g}$ & $55.67 \mathrm{~h}$ & $50.00 \mathrm{j}$ & $68.00 \mathrm{e}$ & $65.00 \mathrm{f}$ & $60.33 \mathrm{~g}$ & $65.00 \mathrm{f}$ \\
\hline ВBCH 51-69 & $73.00 \mathrm{~d}$ & $76.33 \mathrm{c}$ & $70.00 \mathrm{e}$ & $73.00 \mathrm{~d}$ & $95.00 \mathrm{a}$ & $96.67 \mathrm{a}$ & $92.00 \mathrm{~b}$ & $92.33 \mathrm{~b}$ \\
\hline ВВСН 71-89 & $9.00 \mathrm{o}$ & $12.67 n$ & $13.00 \mathrm{n}$ & $12.00 \mathrm{n}$ & $29.00 \mathrm{~m}$ & $31.00 \mathrm{~lm}$ & $30.00 \mathrm{~m}$ & $30.00 \mathrm{~m}$ \\
\hline LSD 0.01 & \multicolumn{8}{|c|}{2.30} \\
\hline
\end{tabular}

Means sharing the same letters within a column or a row are statistically similar $(\mathrm{P}>0.01)$

Table 7. Total number of individuals belonging to Acrididae family trapped by two different sampling methods at different growth stages of rice from southeastern Anatolia region, Turkey during 2010 and 2011

\begin{tabular}{|c|c|c|c|c|c|c|c|c|}
\hline \multicolumn{9}{|c|}{2010} \\
\hline \multirow{2}{*}{ Growth stage } & \multicolumn{4}{|c|}{ D-Vac } & \multicolumn{4}{|c|}{ Sweepnet } \\
\hline & Siverek & Ergani & Hazro & Çinar & Siverek & Ergani & Hazro & Çinar \\
\hline ВBCH 21-39 & $1.00 \mathrm{n}$ & $1.67 \mathrm{mn}$ & $8.00 \mathrm{~g}$ & $13.67 \mathrm{~cd}$ & $4.00 \mathrm{jk}$ & $3.33 \mathrm{j}-\mathrm{m}$ & $8.00 \mathrm{~g}$ & $6.00 \mathrm{hi}$ \\
\hline ВВСН 41-49 & $26.00 \mathrm{a}$ & $3.00 \mathrm{klm}$ & $15.33 \mathrm{c}$ & $23.67 \mathrm{~b}$ & $7.00 \mathrm{gh}$ & $5.00 \mathrm{i}$ & $10.00 \mathrm{f}$ & $27.00 \mathrm{a}$ \\
\hline ВВСН 51-69 & $10.00 \mathrm{f}$ & $2.00 \mathrm{lmn}$ & $2.00 \mathrm{lmn}$ & $11.00 \mathrm{ef}$ & $12.00 \mathrm{de}$ & $3.33 \mathrm{j}-\mathrm{m}$ & $5.00 \mathrm{i}$ & $5.00 \mathrm{i}$ \\
\hline BBCH 71-89 & $2.00 \mathrm{lmn}$ & $5.00 \mathrm{i}$ & $3.00 \mathrm{klm}$ & $7.00 \mathrm{gh}$ & $3.67 \mathrm{jkl}$ & $14.00 \mathrm{c}$ & $2.67 \mathrm{k}-\mathrm{m}$ & $11.00 \mathrm{ef}$ \\
\hline LSD 0.01 & \multicolumn{8}{|c|}{1.79} \\
\hline \multicolumn{9}{|c|}{2011} \\
\hline ВBCH 21-39 & $0.10 \mathrm{~h}$ & $0.10 \mathrm{~h}$ & $0.10 \mathrm{~h}$ & $0.20 \mathrm{~h}$ & $4.00 \mathrm{~g}$ & $4.00 \mathrm{~g}$ & $4.33 \mathrm{~g}$ & $4.00 \mathrm{~g}$ \\
\hline ВBCH 41-49 & $24.00 \mathrm{a}$ & $21.00 \mathrm{~b}$ & $21.00 \mathrm{~b}$ & $23.33 \mathrm{a}$ & $4.00 \mathrm{~g}$ & $5.00 \mathrm{~g}$ & $3.67 \mathrm{~g}$ & $5.00 \mathrm{~g}$ \\
\hline ВBCH 51-69 & $11.00 \mathrm{c}$ & $11.00 \mathrm{c}$ & $8.00 \mathrm{ef}$ & $9.00 \mathrm{de}$ & $10.33 \mathrm{~cd}$ & $7.00 \mathrm{f}$ & $11.00 \mathrm{c}$ & $9.00 \mathrm{de}$ \\
\hline BBCH 71-89 & $1.00 \mathrm{~h}$ & $1.00 \mathrm{~h}$ & $1.00 \mathrm{~h}$ & $1.00 \mathrm{~h}$ & $4.00 \mathrm{~g}$ & $4.00 \mathrm{~g}$ & $5.00 \mathrm{~g}$ & $4.00 \mathrm{~g}$ \\
\hline LSD 0.01 & \multicolumn{8}{|c|}{1.50} \\
\hline
\end{tabular}

Means sharing the same letters within a column or a row are statistically similar $(\mathrm{P}>0.01)$

\section{Discussion}

The current study identified harmful insect species infesting direct seeded rice crop at different growth stages, which will provide valuable insights for the management of these species. A total of 13 species belonging to 4 families were identified, which are regarded as pests of different cultivated crops in earlier studies conducted by different researchers in Turkey. Thisoicetrinus pteroschus (Fischer de Waldheim) has been reported to cause significant damage to vegetables, tobacco and paddy fields in 
Diyarbakir and its vicinity between 1960 and 1961 (Balamir, 1962). Similarly, Euprepocnemis plorans (Charpentier) is the most dangerous pest species of maize and cereals (Şimsek, 1988). Platycleis escalerai Bolívar, Calliptamus barbarus FisherWaldheim and Aiolopus strepens (Latr.) have been reported as harmful pests in numerous crops (Karabă̆ et al., 1971; Karabağ, 1975; Çıplak et al., 1991, 1996). Different studies have reported that different orthopteran species, i.e, Gryllo gryllotalpa (L.), T. pterostichus, Locusta migratoria migratorioides (R. \& F.) and Conocephalus discolor (Thunberg) are noxious pests of rice crop (Alkan, 1948; Balamir, 1962; Uzunali, 1976; Serel, 1978). The identified insect species in the current study infest rice crop at different growth stages. Therefore, management strategies/efforts should be opted according to the infestation time of harmful insect species.

Different growth stages of rice crop, sampling methods and surveyed rice fields significantly differed for insect infestation. It is known that the life cycle of many insect pest species is closely linked with the development of the rice plant (Reissig et al., 1986) and the differences in the insect density at different growth stages of rice crop are owed to the feeding preference, life form and damaging habit of the insect species (Reissig et al., 1986; Pathak and Khan, 1994). Similarly, the differences among different surveyed fields are thought to be the result of micro environmental conditions (Table 1), weed infestation and site-specific management practices opted by the farmers (Reissig et al., 1986; Pathak and Khan, 1994). Likewise, the differences in the efficacy of different sampling methods for trapping insect specises can be explained with the size, weight and life stage of the insect species (Reissig et al., 1986). The D-Vac sampling method usually sucks the insect which are smaller sized, lighter in weight and at the most mobile stage, whereas the sweep net method traps heavier insects, relatively less mobile and bigger in size (Turnipseed, 1974; Kogan and Herzog, 2012). Therefore, the selection of sampling methods should be based on the target insect species and growth stage of the crop.

Crane fly (Tipulidae) species infested the rice crop during early growth season, i.e., BBCH 21-39 growth stage during both years of the study. The highest density of crane fly species at early growth stage is directly linked to their damage caused to the rice crop. The larvae of crane fly species feed on pastures and spring cereals, roots and young seedlings of rice crop (Rao et al., 2006; Duman et al., 2014). The larval movement below soil uproots the emerging rice seedling leading to plant mortality (Duman et al., 2014). Therefore, the highest density of crane fly species at initial growth stage can be linked with the damaging habit, life cycle and growth stage of the species. The identified crane fly species (Tipula orientalis) is a widespread cosmopolitan species frequently found near streams and in cultivated wheat and rice fields (Duman et al., 2014). Rice is cultivated through direct seeding method in southeastern Anatolia region, and the irrigation applied to direct-seeded rice creates favorable environment for the development of $T$. orientalis larvae. Thus, sowing method of rice in the region is another reason of higher density of crane fly. The lowest density of crane fly species at later growth stages of rice crop can be explained with the life cycle of the pest. The pest completes its life cycle and switches to diapause at later growth stages of rice due to which the lowest number of insects were trapped at $\mathrm{BBCH}$ 71-89 growth stage of rice crop. Xingquan et al. (1993) stated that cold swamps and meadows are the primary habitats of Tipula sp and the larva of the species cause highest damage at the end of 
May. The current results of the highest density of Tipulidae species at initial growth stage of rice crop are like the findings of Qian (1982) and Xingquan et al. (1993).

Thrips species had the highest infestation at different growth stages of rice crop in 1 st and 2nd year of the study. The highest density was recorded at maturity (BBCH 7189) during 1st year of the study, whereas the highest density was noted at BBCH 21-39 during 2nd year. The reasons for the differences in the density of thrips species at different growth stages in different years can be explained by the prevailing climatic conditions and weeds' infestation at different growth stages of rice crop. The farmers of southeastern Anatolia region stop irrigation at $\mathrm{BBCH} 71-89$ growth stage of rice whereas temperature is high compared to the rest of the growth stages. The increased temperature coupled with no irrigation decreases relative humidity, thus, creating favorable conditions for the population development of thrips species. Thrips outbreaks are frequently reported during dry periods in rice crop (Hsu et al., 1978; Pathak and Khan, 1994). It is well known that thrips species mostly live in flowers, but many species complete their life cycle on leaves (Ananthakrishnan, 1993; Trdan et al. 2005; Van Haperen et al., 2019). A few of thrips species are predatory and probably about half of thrips species feed on fungi (Palmer et al., 1989). The damage caused to plants by thrips through feeding or egg laying is often ascribed to other pest species. Two thrips species were detected in the current study and discoloration on the leaves was noted during seedling stage. Besides feeding damage was also recorded during heading stage of rice (Hsu et al., 1978). Stenchaetothrips biformis (Bagnall) and Haplothrips aculeatus (Fabricius) have been recorded throughout rice growth season; however, these species are more abundant during the seedling and flowering stages of rice in some Asia countries (Madhusudhan and Gopalan, 1989; Pathak and Khan, 1994). The population declines in early September and disappears by mid-September (Pathak and Khan, 1994). Like the results of the first year of the current study, thrips infestation is generally recorded during the first week of August, with peak infestation during the second and third weeks in India. Hsu et al. (1978) reported that Baliothrips biformis (Bagn.) infests rice crop at 3-4-leaf seedling and tillering stages, whereas Frankliniella tenuicornis (Uzel) and Haplothrips aculeatus (F.) at late head-bearing and heading stages. These findings support the observation of current study.

Grasshopper species (Tettigonidae and Acrididae) had the highest density at BBCH 41-49 and BBCH 51-69 growth stages of rice crop. These findings are line with the results of Serel (1978) reported that grasshoppers appear in rice crop during first week of July. These results indicated that grasshopper species infest rice crop during mid to late growth season. Due to this reason, rice farmers use insecticides to control grasshopper species during July. However, the farmers in southeastern Anatolia region do not use any control measures against grasshoppers in rice crop. The insecticides are occasionally used to control grasshoppers in the region once the densities of grasshopper species are high. The higher density of grasshopper species observed in the current study is because the selected rice fields were closer to overwintering/migration areas of grasshoppers. The results obtained in the current study are similar to the results of several earlier studies reporting the identified insect species as harmful pests of rice crop (Balamir, 1962; Karabağ et al., 1971; Karabağ, 1975; Serel, 1978; Memişoğlu et al., 1986; Şimşek, 1988; Çıplak and Demirsoy, 1991; Çıplak et al., 1996; Satar and Özbay, 2003). 


\section{Conclusion}

The current study concludes that different insect species infest direct seeded rice crop at different growth stages in southeastern Anatolia region of Turkey; however, actual losses caused by these species are still unknown. The studies relating to the losses caused by the identified insect species in the region are needed. It is also concluded that the differences in density of the insect species at different growth stages and in different fields necessitate site-specific and growth stage-specific management practices for the identified insect species in the region. Moreover, different sampling methods can be used for trapping harmful insect species (based on their size, weight and growth stage etc.) at different growth stages of rice.

Acknowledgements. The authors express their sincere gratitude to Commission of General Directorate of Agricultural Researches and Policies (TAGEM) for funding this study under project number TAGEMB10/07-04(01-02)-08. We would like to thank several rice farmers who allowed the authors to conduct this research in their cultivated fields. Authors also express sincere thanks to Mahsum DEMIR and Deniz ÇAPLIK for their assistance in field selection and technical support.

\section{REFERENCES}

[1] Alkan, B. (1948): Cereal Pests in Central Anatolia. - Ankara University Publication, Ankara. 132 pp.

[2] Ananthakrishnan, T. N. (1993): Bionomics of thrips. - Annual Review of Entomology 38(1): 71-92.

[3] Balamir, S. (1962): Türkiye'de Son Yıllarda Zararı Görülen Bir Çekirge Türü Thisocertrinus pterostichus F.W. - Plant Protection Bulletin 2(2): 3-10.

[4] Binns, M. R., Nyrop, J. P. (1992): Sampling insect populations for the purpose of IPM decision making. - Annual Review of Entomology 37(1): 427-453.

[5] Buffington, M. L., Redak, R. A. (1998): A comparison of vacuum sampling versus sweep netting for arthropod biodiversity measurements in California coastal sage scrub. Journal of Insect Conservation 2(2): 99-106.

[6] Castro, D. C. (1970): Rice Production Manual. - Los Banos: University of Philippines/IRRI, University of Philippines/IRRI.

[7] Catling, D. (1992): Rice in Deep Water. - London: MacMillan Press Ltd.; 1992.

[8] Çetin, S. C., Karaca, A., Haktanır, K., Yildiz, H. (2007): Global attention to Turkey due to desertification. - Environmental Monitoring and Assessment 128(1-3): 489-493.

[9] Çıplak, B., Demirsoy, A. (1991): Arguvan (Malatya) ve Çevresinde Orthoptera (Ins.) Faunasının İncelenmesi. - Turkish Journal of Zoology 15: 98-114.

[10] Çıplak, B., Demirsoy, A., Bozcuk, A. N. (1996): Malatya ve civarı Caelifera (OrthopteraInsecta) Faunas1. - Turkish Journal of Zoology 20: 17-31.

[11] Duman, M., Mutlu, C.. (2011): Determination of Harmful and Beneficial Insect in the Karacadağ Rice Variety Cultivated Area. - Proceedings of the Fourth Turkish National Congress of Entomology, 28-30 June 2011, Kahramanmaras, Turkey. 287p.

[12] Duman, M., Mutlu, Ç., Büyük, M., Karaca, V. (2013): Beneficial insects, spider and polinater species determined in the Karacadağ paddy growing areas. - Turkish Journal of Biological Control 4(1): 53-64.

[13] Duman, M., Mutlu, Ç., Büyük, M. (2014): A new pest in rice growing fields in Southeastern Anatolia: Tipula orientalis Laskschewitz (Diptera: Tipulidae). - Turkish Bulettin of Entomology 4(2): 67-77.

[14] FAOSTAT. (2019): The Food and Agriculture Organization, FAO. http://www.fao.org/faostat/en/\#home (Date accessed: 28.03.2019). 
[15] Horgan, F. G., Crisol Martinez, E., Almazan, M. L. P., Romena, A., Ramal, A. F., Ferrater, J. B. (2016): Susceptibility and tolerance in hybrid and pure-line rice varieties to herbivore attack: biomass partitioning and resources-based compensation in response to damage. - Annals of Applied Biology 169: 200-213.

[16] Horgan, F. G. (2017): Insect Herbivores of Rice: Their Natural Regulation and Ecologically Based Management. - In Rice Production Worldwide pp. 279-302, Springer, Cham.

[17] Horgan, F. G., Ramal, A. F., Villegas, J. M., Almazan, M. L. P., Bernal, C. C., Jamoralin, A., Arroyo, C. (2017): Ecological engineering with high diversity vegetation patches enhances bird activity and ecosystem services in Philippine rice fields. - Regional Environmental Change 17(5): 1355-1367.

[18] Hsu, Z. Y., Li, J. D., Chang, C. H., Hu, Z. C. (1978): Studies on the thrips infesting rice in Jin-ping, Kweichow Province. - Acta Entomologica Sinica 21(1): 13-26.

[19] IBM. (2012): SPSS Statistics for Windows v. 20. - 1-8. Armonk, NY: IBM Corporation.

[20] Islam, Z., Karim, A. N. M. R. (1997): Whiteheads associated with stem borer infestation in modern rice varieties: an attempt to resolve the dilemma of yield losses. - Crop Protection 16: 303-311.

[21] Karabağ, T., Gümüşsuyu, İ., Balamir, S., Tutkun, E. (1971): Orthoptera Fauna of Turkey (II). - Plant Protection Bulletin 2(2): 73-100.

[22] Karabağ, T. (1975): Studies in the Turkish Orthoptera (Ins.) I New Species and Less Known Tettigoniidae. - Journal of Natural History 9: 337-350.

[23] Kiritani, K. (1979): Pest management in rice. - Annual Review of Entomology 24: 279312.

[24] Kogan, M., Herzog, D. C. (2012): Sampling methods in soybean entomology. - Springer Science \& Business Media. 596 pp.

[25] Lancashire, P. D., Bleiholder, H., Boom, T. V. D., Langeluddeke, P., Stauss, R., Weber, E., Witzenberger, A. A. (1991): Uniform decimal code for growth stages of crops and weeds. - Annals of Applied Biology 119: 561-601.

[26] Madhusudhan, V. V., Gopalan, M. (1989): Studies on the biology of rice thrips, Stenchaetothrips biformis (Bagnall) in India. - Tropical Pest Management 35: 394-396.

[27] Meier, U. (1997): Growth stages of mono-and dicotyledonous plants. - Federal Biological Research Centre for Agriculture and Forestry.

[28] Memişoğlu, H., Özkan, M., Melan, K. (1986): Faunistic Survey Studies in the Rice Fields in Central Anatolia. - Plant Protection Bulletin 26(3-4): 97-111.

[29] Muthayya, S., Sugimoto, J. D., Montgomery, S., Maberly, G. F. (2014): An overview of global rice production, supply, trade, and consumption. - Annals of the New york Academy of Sciences 1324(1): 7-14.

[30] Mutlu, Ç., Sertkaya, E., Güçlü, Ş. (2008): Determination of Cicadellidae (Homoptera) species in second crop maize and their distribution in Diyarbakır province of Turkey. Turkish Journal of Entomology 32(4): 281-301.

[31] Mutlu, Ç., Duman, M., Karaca, V., Bayram,Y., Süer, İ. E. (2016): he species of Cicadellidae, Cixiidae and Delphacidae (Hemiptera) on Karacadag rice and the effects of weeds on their populations. - Turkish Bulletin of Entomology 6(4): 279-289.

[32] Nik Mohammad Noor, N. S., Chang, P. M. (1995): Rice leaffolder in Malaysia and implications of not controlling them. - International Rice Research Institute. Proceedings of the workshop on reducing early season insecticide use for leaffolder control in rice: impact, economics and risks, Los Baños, pp. 1-15.

[33] Palmer, J. M., Mound, L. A., du Heaume, G. J. (1989): CIE guides to insects of importance to man 2. - Thysanoptera. CAB Int, Wallingford, pp 73.

[34] Pathak, M. D., Dyck, V. A. (1973): Developing an integrated method of rice insect pest control. - PANS Pest Articles \& News Summaries 19(4): 534-544.

[35] Pathak, M. D., Khan, Z. R. (1994): Insect Pests of Rice. - International Rice Research Institute, Los Baños. 
[36] Qian, K. C. (1982): A preliminary study on the biology of Tipula aino Alexander. Insect Knowledge 19(5): 9-11.

[37] Rao, S., Liston, A., Crampton, L., Takeyasu, J. (2006): Identification of larvae of exotic Tipula paludosa (Diptera: Tipulidae) and $T$. oleracea in North America using mitochondrial cytB sequences. - Annals of the Entomological Society of America 99(1): 33-40.

[38] Reissig, W. H., Heinrichs, E. A., Litsinger, J. A., Moody, A., Fiedler, L., Mew, T. W, Barrion, A. T. (1986): Illustrated Guide to Integrated Pest Management in Rice in Tropical Asia. - International Rice Research Institute, Los Baños.

[39] Rubia, E. G., Heong, K. L., Zalucki, M., Gonzales, B., Norton, G. A. (1996): Mechanisms of compensation of rice plants to yellow stem borer Scirpophaga incertulas (Walker) injury. - Crop Protection 15: 335-340.

[40] Satar, A., Özbay, C. (2003): On the Orthoptera (insecta) Fauna of the Karacadag Mountains and the Tigris Basin (Diyarbakir,Turkey). - Boletín SEA 32: 115-120.

[41] Schotzko, D. J., O'Keeffe, L. E. (1989): Comparison of Sweep Net., D-Vac., and Absolute Sampling., and Diel Variation of Sweep Net Sampling Estimates in Lentils for Pea Aphid (Homoptera: Aphididae), Nabids (Hemiptera: Nabidae)., Lady Beetles (Coleoptera: Coccinellidae)., and Lacewings (Neuroptera: Chrysopidae). - Journal of Economic Entomology 82(2): 491-506.

[42] Serel, İ. (1978): Güneydoğu Anadolu Bölgesinde çeltik zararlılarının tanınmaları, yayılış alanları ve ekonomik önemleri üzerine araştırmalar. - (Ph.D Thesis) 92. Ege University. Agriculture Faculty, İzmir, Turkey. (In Turkish).

[43] Settle, W. H., Ariawan, H., Astuti, E. T., Cahyana, W., Hakim, A. L., Hindayana, D., Estari, A. S. (1996): Managing tropical rice pests through conservation of generalist natural enemies and alternative prey. - Ecology 77: 1975-1988.

[44] Steel, R. G. D., Torrie, J. H., Dickey, D. A. (1997): Principles and Procedures of Statistics: A Biological Approach. - New York: McGraw-Hill.

[45] Şimşek, Z. (1988): Studies on the Insect Species Harmful on Corn and Sorghum, Their Determination and Distribution in Eastern and Southeastern Anatolia Regions in Turkey. - Diyarbakir Plant Protection Research Institute, Series of Research Works No: 6, 87 p.

[46] Teoman, A., Kavut, H. (1979): Studying on insect fauna in the paddy fields of the Aegean Region. - Annals of Plant Protection Research 14: 10-12. İzmir, Turkey.

[47] Trdan, S., Andjus, L., Raspudić, E., Kač, M. (2005): Distribution of Aeolothrips intermedius Bagnall (Thysanoptera: Aeolothripidae) and its potential prey Thysanoptera species on different cultivated host plants. - Journal of pest science 78(4): 217-226.

[48] TURKSTAT. (2016): Turkish Statistical Institute. - www.tuik.gov.tr/Start.do. (Date accessed: December 2018).

[49] Turnipseed, S. G. (1974): Sampling soybean insects by various D-Vac, sweep, and ground cloth methods. - Florida entomologist 57(3): 217-223.

[50] Uzunali, S. (1976): Karadeniz Bölgesi Çeltiklerinde (Oryza sativa L.) zarar yapan önemli böcek türleri, tanınmaları, yayılış alanları ve zararları üzerinde araştırmalar. - (Ph.D thesis) 68 pp. Ege University Agriculture Faculty, İzmir, Turkey. (In Turkish).

[51] Van Haperen, P., Voorrips, R. E., Van Loon, J. J., Vosman, B. (2019): The effect of plant development on thrips resistance in Capsicum. - Arthropod-Plant Interactions 13(1): 1118.

[52] Way, M. J., Heong, K. L. (1994): The role of biodiversity in the dynamics and management of insect pests of tropical irrigated rice - a review. - Bulletin Entomological Reserach 84: 567-587.

[53] Xingquan, Y., Jianhua, W., Taifang, W. (1993): Studies on the bionomics of Tipula sp. and its control. - Entomological Knowledge 30(1): 4-6.

[54] Yiğit, A., Doğanlar, M., Sertkaya, E., Evren, N. (2003): Comparison of two method used in sampling of sunn pest, Eurygaster integriceps Puton, (Het.; Scutelleridae) in Şanlıurfa, Turkey. - GAP III. Agriculture Congress, 02-03 November, Şanlıurfa, Turkey. 
[55] Y1lmaz, E., Karsavuran, Y. (2009): Fluctuations of Zyginidia pullula (Boheman, 1845) and Asymmetrasca decedens (Paoli) (Hom.: Cicadellidae) Populations in Maize Cultivation Areas of İzmir Province. - Proceedings of the Third Plant Protection Congress of Turkey, 15-18 July, Van, Turkey.

[56] Yürüten, O., Günaydın, T., Ersoy, G. (1982): Marmara Bölgesi çeltik tarlalarında zararlı ve faydalı fauna sürveyi. - Project Final Report (In Turkish). Plant Protection Research Institue, Istanbul, Turkey. 